\title{
Epitaxial thin films grown by pulsed laser deposition
}

\author{
Dave H.A. Blank \\ University of Twente, Faculty of Science and Technology, and MESA ${ }^{+}$Institute for Nanotechnology \\ P.O. Box 217, 7500 AE The Netherlands
}

\begin{abstract}
Pulsed Laser Deposition (PLD) is attractive for research on epitaxial grown films because it is fast and one can easily investigate a wide range of different materials and compositions. Currently, a major issue in the growth of oxide materials with PLD is the control of the surface morphology. For most materials it is necessary to control the thickness and roughness of the thin films down to an atomic scale. Such well-controlled growth can also be used to manufacture artificially layered structures of different materials with different properties. In this way it is possible to create a whole new class of materials. It would be possible to create materials tailor-made to applications. Such materials are also ideal for the purpose of understanding the physics and the search for materials with even yet not-known properties.
\end{abstract}

The flexibility of the pulsed laser deposition set-up offers the researcher a large freedom of choice in target material, ablation characteristics, target-substrate geometry, ambient gas and its pressure, and substrate temperature. All these parameters influence plasma formation, film growth, and film properties. Deposition parameters can be varied over several orders of magnitude. Especially the ambient pressure is known for its large dynamic range of more than seven orders of magnitude. Furthermore, the kinetic energies of species arriving at the substrate surface can be tuned over three orders of magnitude $(0.1-400 \mathrm{eV})$. The large dynamic ranges of deposition parameters result in different regimes of ablation and deposition. Due to powerful lasers with short pulse lengths, high laser power densities can be realized. This makes the ablation of a wide range of materials possible.

Pulsed laser deposition has become the workhorse for the development of complex oxide materials. Much effort is put in the deposition of excellent textured layers without grain boundaries. In general the properties of highly oriented films approximate the properties of single crystals. Single or multi-layer structures require a well-conditioned process technique. The deposited layers must have a large homogeneity with well-defined material properties, smooth surfaces, and, in the case of oxides, the correct oxygen stoichiometry. Important is the possibility to combine PLD with standard in-situ diagnostic techniques. Growth monitoring became possible even at relative high deposition pressures using ellipsometry and so-called high pressure Reflection High Energy Electron Diffraction (RHEED). These developments have helped to make PLD a grown-up technique to fabricate complex materials and structures.

The recent developments in the strongly correlated materials, in particularly the metal oxides, have brought on many inventive ideas to apply these materials in novel device concepts. During the last decade a tremendous progress has been made in controlling these complicated materials. To name a few, these are the epitaxial growth technique, understanding of the properties of their defect structure, atomic-level control of their layering, in the case of oxides the manipulation of the oxygen contents and dopant densities, etc.. With our development of pulsed laser deposition with control at an atomic level using high-pressure reflective high-energy electron diffraction we are able to control the growth of these materials and to introduce new growth manipulations, like pulsed laser interval deposition. At present, extremely sharp and homogeneous interfaces can be realized and this is, for example, yet utilized in magnetic tunnel junctions in the ferromagnetic 'bad metal' $\mathrm{SrRuO}_{3}$. In this presentation I like to show these unique techniques and their use the obtained results to design complex materials 'on demand'.

Structures can be built with atomic precision and possess properties that surpass those of the individual building blocks. It also underlines the potential of designing artificial superlattices with unique properties. For example, most ferromagnetic materials show no ferroelectricity. But what would a superlattice made from ferroelectric and ferromagnetic building blocks be like? Would it show ferroelectric or magnetic behaviour, or have a mix of properties? New phenomena might even emerge. Here, then, is an invitation to materials scientists to design and build their own superlattice*.

*Guus Rijnders and Dave H.A. Blank, Nature News \& Views 433 (2005) 